\title{
From High Electron Mobility \\ GaN/AlGaN Heterostructures to Blue-Violet InGaN Laser Diodes. Perspectives of MBE for Nitride Optoelectronics
}

\author{
C. SkIERBISZEWSKI* \\ Institute of High Pressure Physics, PAS \\ Sokołowska 29/37, 01-142 Warsaw, Poland
}

\begin{abstract}
The recent progress in growth of nitride based semiconductor structures made by plasma assisted MBE is presented. This technology is ammonia free and nitrogen for growth is activated in RF plasma source from nitrogen molecules. The new growth mechanism - adlayer enhanced lateral diffusion of adatoms on semiconductor surface is studied in plasma assisted MBE. This mechanism enables us to achieve high quality step-flow epitaxy at temperatures $600-750^{\circ} \mathrm{C}$, much lower than expected from classical estimates based on the melting point of GaN. We show that growth at low temperatures in metal rich (gallium or indium) regime, together with use of low dislocation bulk GaN substrates, results in high quality of (In, Al, Ga)N layers and sharp interfaces. We demonstrate record high mobility of two-dimensional electron gas at GaN/AlGaN interface (with mobility exceeding $100000 \mathrm{~cm}^{2} /(\mathrm{V} \mathrm{s}$ ) at $4.2 \mathrm{~K}$ and $2500 \mathrm{~cm}^{2} /(\mathrm{V} \mathrm{s})$ at $\left.300 \mathrm{~K}\right)$ and report on first blue-violet InGaN multiquantum well laser diodes, operating in $407-422 \mathrm{~nm}$ wavelengths range. In this paper, we discuss also properties of strain compensated InAlN/InGaN multiquantum wells grown by plasma assisted MBE which are very attractive for telecommunication applications at $1.5 \mu \mathrm{m}$ wavelengths like electro-optical modulators or all-optical switches.
\end{abstract}

PACS numbers: 42.55.Px, 85.35.Be, 42.60.By, 73.21.Cd

\section{Introduction}

For many years molecular beam epitaxy (MBE) was regarded to be less adopted than metal-organic vapor phase epitaxy (MOVPE) for growth of nitrides $[1,2]$. In spite of MBE's many potential advantages, such as much better qual-

*e-mail: czeslaw@unipress.waw.pl 
ity of interfaces, sharper doping profiles and superior in situ growth monitoring capabilities [3-5], the considerably inferior optical quality of grown layers, when compared to similar structures grown by MOVPE, fueled widespread belief that this technique would never become a viable alternative in the field of GaN based optoelectronics. Only very recently the room temperature, pulsed operation of MBE-grown laser diodes (LDs) with $400 \mathrm{~nm}$ emission wavelength was demonstrated [6]. These devices were grown with so-called ammonia MBE [3], where atomic beams are used for group III elements, while $\mathrm{NH}_{3}$ is used as the nitrogen precursor. Use of ammonia gas flows of $\approx 200 \mathrm{sccm}$, high V/III flux ratios (of up to $10^{3}$ ), high growth temperatures up to $950^{\circ} \mathrm{C}$, bis-cyclopentadienylmagnesium $\left(\mathrm{Cp}_{2} \mathrm{Mg}\right)$ as a source of $\mathrm{Mg}$, shift the growth conditions for this technique very close to those applied in the nitride MOVPE reactors.

In spite of its potential advantages for both optoelectronic [3] and electronic [7] devices, ammonia MBE has not been the technique of choice for the majority of nitride MBE community. Indeed, corrosive nature of ammonia compounded by its large flows creates additional hazards and technological challenges as well as leads to high hydrogen background during the epitaxial process. In the more widely employed, so-called plasma assisted MBE (PAMBE) [5], purified nitrogen gas is activated using an RF-plasma and supplied to the growing surface at typical flow rates of 1-2 sccm. With notable exception of the growth on the bulk substrates [8], high quality epitaxial layers have been demonstrated only for the (0001) Ga-polarity surface, much as for ammonia MBE. However, early experimental results showed [9] that unlike ammonia MBE, PAMBE requires group III-rich conditions to achieve good material quality. Much progress has been made in both theoretical $[10,11]$ and experimental $[12,13]$ understanding of the growth kinetics for such metal rich conditions. Perhaps the most insightful were recent results of modeling based on the density-functional theory which revealed existence of a very efficient lateral diffusion channel for adatoms on semiconductor surface just below the thin metallic film [10]. Surprisingly small activation energies for this adlayer enhanced lateral diffusion (AELD) enable high quality step-flow epitaxy at temperatures much lower than expected from classical estimates based on the melting point of the grown material. Indeed, in spite of the relatively low growth temperatures, $650-750^{\circ} \mathrm{C}$, state of the art $\mathrm{GaN} / \mathrm{AlGaN}$ heterostructures with record high mobilities of two-dimensional electron gas [14-17] were grown with PAMBE, making it the technique of choice for the production of electronic devices. Also, very recently, a considerable improvement in the quality of PAMBE-grown LEDs and LDs was reported $[18,19]$, signaling usefulness of this technique also for the optoelectronic devices.

\section{Growth kinetics in PAMBE}

In general, basing on thermodynamical considerations, to ensure enough high lateral diffusion for impinging adatoms (and to achieve high quality step flow 
growth mode), the growth temperature $T_{\mathrm{G}}$ should be about half of the temperature of melting point $T_{\mathrm{M}}$ of grown material. As an example we can compare GaAs and GaN. For GaAs $T_{\mathrm{M}}$ is $1240^{\circ} \mathrm{C}$ while for $\mathrm{GaN}$ it is in the range between $2270^{\circ} \mathrm{C}$ (experimentally determined for $6 \mathrm{GPa}[20]$ ) and $2530^{\circ} \mathrm{C}$ (theoretically calculated [21]). It is experimentally demonstrated that for GaAs, the high quality layers are achieved for growth temperatures above $560^{\circ} \mathrm{C}$. For GaN growth temperature of $1050-1100^{\circ} \mathrm{C}$ is used for growth of GaN in MOVPE systems with ammonia overpressure in the range of $0.2-1$ bar. There is no intrinsic limitation to heat GaN substrate to temperature $1100^{\circ} \mathrm{C}$ in the $\mathrm{MBE}$ system, however due to the vacuum conditions, GaN starts to decompose at much lower temperatures, around $800^{\circ} \mathrm{C}$, which may prevent a successful growth in MBE. Due to the low diffusion of $\mathrm{N}$ atoms on the GaN surface, the early efforts to grow GaN in MBE at temperatures below $800^{\circ} \mathrm{C}$ gave unsatisfactory results. It was believed also that MBE can give good results only if the growth temperature is close to the MOVPE conditions which requires a high partial pressure over the substrate.

The breakthrough in study of growth kinetics in PAMBE came with finding that in Ga-rich conditions it is possible to grow relatively smooth layers at low growth temperatures. However, in such conditions it was easy to create Ga droplets on GaN surface and high quality material was mainly formed in the regions between droplets. Further study of Ga auto-surfactant effect led to conclusion that it is important to use very well defined amount of excess Ga (just below value where Ga droplets are formed on GaN surface) for successful two-dimensional step flow growth mode [12-14]. From the technical point of view, the amount of excess Ga, $\Phi_{\mathrm{Ga}}^{\mathrm{EXESS}}$, required for high quality growth was found to depend strongly on the growth temperature with activation energy, $E_{\mathrm{A}}$, around $2.8 \mathrm{eV}$, close to the activation energy of desorption of Ga atoms from liquid gallium: $\Phi_{\mathrm{Ga}}^{\mathrm{EXCESS}}=\Phi_{0} \exp \left(-E_{\mathrm{A}} / k T\right)[12]$.

Microscopic mechanism of enhanced mobility of atoms for Ga-rich conditions on GaN surface is following: when gallium forms bi-layer coating on Ga-polarity side of GaN, impinging $\mathrm{N}$ atoms penetrate easily this layer and new diffusion channel for $\mathrm{N}$ atoms is formed just below Ga layer (see Fig. 1). This so-called AELD channel increases dramatically the lateral mobility of $\mathrm{N}$ atoms and helps them to find nearest atomic step in a GaN surface (Fig. 1). This finding is also valid for In coating. When GaN is coated by metal (Ga or In) the barrier for lateral diffusion for nitrogen atoms is reduced from $1.3 \mathrm{eV}$ to about $0.5-0.3 \mathrm{eV}$ [10]. It means that high quality growth of GaN is possible at relatively low temperatures.

All nitride structures presented in this paper were grown in custom designed V90 Oxford MBE system. Ultra-high purity gas handling manifold together with Veeco UniBulb RF Plasma cell was used as a source of activated nitrogen. Two types of substrates were used: (a) MOCVD GaN/ $/ \mathrm{Al}_{2} \mathrm{O}_{3}$ templates and (b) bulk GaN substrates. The epi-ready bulk substrates were prepared in the three-step process of mechano-polishing, dry etching and deposition of $2 \mu \mathrm{m} \mathrm{GaN}$ :Si buffer 


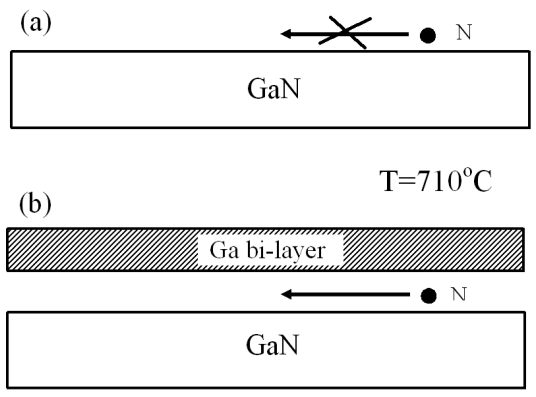

Fig. 1. The $\mathrm{N}$ adatom kinetics on $\mathrm{GaN}$ (Ga polarity) surface at $710^{\circ} \mathrm{C}$. Without Ga coating high barrier for lateral $\mathrm{N}$ diffusion leads to 3D growth mode (a). With bi-layer Ga coverage - effective lateral diffusion channel below $\mathrm{Ga}$ is opened for step-flow 2D growth mode (b).

layer in the MOVPE reactor. The back sides of the substrates were coated with $0.7 \mu \mathrm{m}$ of molybdenum layer to improve the thermal coupling for radiative heating. Special holders capable of accommodating small irregularly shaped substrates $\left(5 \times 5\right.$ to $10 \times 10 \mathrm{~mm}^{2}$ approximate size $)$ and designed to minimize edge effects ensured high temperature uniformity across the entire substrate area. For growth on polar substrates, Ga polarity face was used.

Figure 2 gives an insight how surface morphology depends on the choice of $\mathrm{Ga}$ and $\mathrm{N}$ fluxes at constant growth temperature. We present the data for slightly nitrogen-rich conditions (squares) and for Ga-rich conditions (dots). For the low Ga fluxes $\left(\Phi_{\mathrm{Ga}} \sim \Phi_{\mathrm{N}}\right)$ three-dimensional growth mode is observed leading to the GaN morphology shown in Fig. 3a. For the Ga fluxes higher than $\mathrm{N}$ three regions can be found. First, where mixed 2D and 3D growth is observed - very flat surfaces related with $2 \mathrm{D}$ growth can be found which indicates that probably bilayer Ga coating is formed only locally (Fig. 3b). Figure 3c shows picture of $2 \mathrm{D}$ growth mode achieved when bilayer coating of $\mathrm{Ga}$ is formed on entire substrate. For higher Ga flux, the Ga droplets formed on the surface. We would like to stress here that AELD channel allows to achieve high mobility of adatoms at low temperatures in lateral direction. In direction perpendicular to the surface the low growth temperature stops interdiffusion. This finding is very important to achieve very sharp interfaces between different nitride alloys which lead to superior device performance.

The local density approximation (LDA) calculations for $\mathrm{N}$ adatom diffusion barrier height below metal layer were performed for ideal GaN substrate. If defects are present in substrate it can increase the diffusion barrier [10]. Therefore use of the high quality GaN bulk substrates can promote high quality growth at very low growth temperatures. The progress in synthesis of high pressure GaN bulk crystals at the Institute of High Pressure Physics, PAS [22] allowed us for systematic study of growth mechanisms on those substrates. 


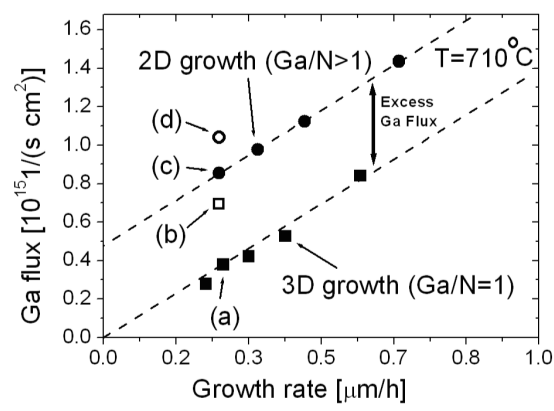

Fig. 2. Growth diagram of GaN for $T=710^{\circ} \mathrm{C}$. Dots - data for $\mathrm{Ga} / \mathrm{N}>1$ where $2 \mathrm{D}$ step-flow mode is observed. Squares - data for $\mathrm{Ga} / \mathrm{N} \approx 1$ (3D growth mode). Open square and circle show the growth in mixed (2D and $3 \mathrm{D})$ growth mode and 2D growth mode with Ga droplets, respectively. Morphology of samples (a)-(d) are presented in Fig. 3.

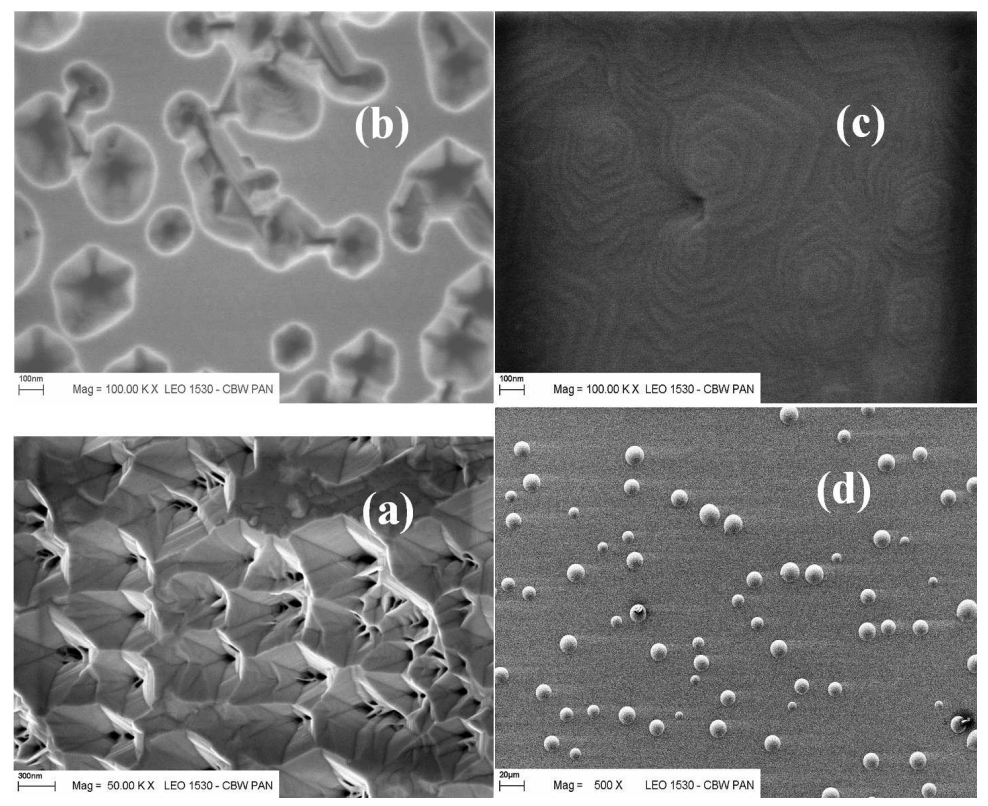

Fig. 3. The scanning electron microscope images of GaN grown on GaN/sapphire templates by PAMBE at constant temperature and constant nitrogen flux with different Ga fluxes. (a) $\mathrm{Ga} / \mathrm{N}=1,3 \mathrm{D}$ growth mode. (b) $\mathrm{Ga} / \mathrm{N}=1.8,3 \mathrm{D}$ and $2 \mathrm{D}$ mixed growth mode. (c) $\mathrm{Ga} / \mathrm{N}=2.4,2 \mathrm{D}$ step-flow growth mode. (d) $\mathrm{Ga} / \mathrm{N}=2.8,2 \mathrm{D}$ growth mode and Ga droplets on the surface.

In Fig. 4 we present comparison of surface morphology of GaN layer grown by PAMBE on GaN bulk crystal (Fig. 4a) and GaN/sapphire template (Fig. 4b). The clear atomic steps are present on GaN layer grown on bulk while dislocation 

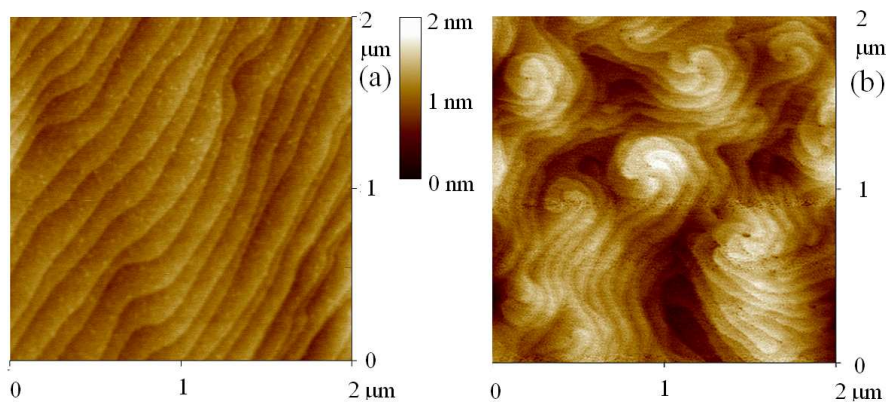

Fig. 4. Atomic force microscope images of the GaN layers on bulk crystal (a) and GaN/sapphire template (b).

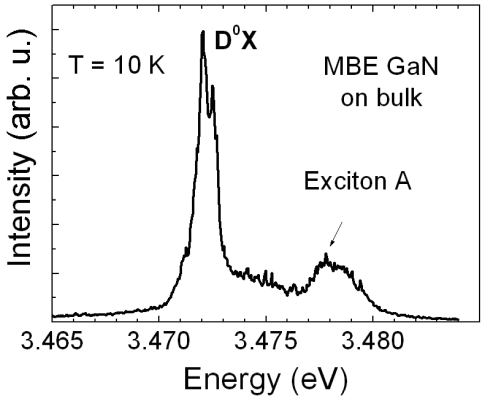

Fig. 5. Low temperature photoluminescence from GaN layer grown by PAMBE.

mediated step-flow growth mode is observed for GaN/sapphire substrate. The quality of homoepitaxial GaN layer is confirmed by photoluminescence experiments at low temperatures where sharp line originating from donor bound exciton, free exciton was found (Fig. 5) [16].

\section{Two-dimensional electron gas on GaN/AlGaN interface}

Properties of two-dimensional electron gas (2DEG) in GaN/AlGaN interface are currently intensively studied due to the great impact of GaN based devices for electronic applications. It was already shown that due to the piezoelectric doping mechanism, the concentration of electrons in 2DEG is controlled by the thickness and $\mathrm{Al}$ content of AlGaN layer grown on Ga-polarity side [13, 23, 24]. A very high electron concentration of $2 \mathrm{DEG}, n_{2 \mathrm{D}}$, of the order of $10^{13} \mathrm{~cm}^{-2}$ obtained without additional modulation doping together with the mobility, $\mu_{2 \mathrm{D}}$, of about $1000-2000 \mathrm{~cm}^{2} /(\mathrm{V} \mathrm{s})$ at the room temperature (RT), makes GaN/AlGaN heterostructures to be very promising for high power and high frequency transistors [23].

From the technological point of view, the main factor limiting the performance of the high power optoelectronic and electronic devices is the presence 
of different types of dislocations, mainly due to the growth of GaN on foreign substrates with a large lattice mismatch such as sapphire or SiC. For electronic applications of field effect transistors at room temperatures and above the RT, an increase in the product of the electron mobility and concentration, i.e., the conductivity is a figure of merit. With regard to the RT, it was not long ago that the maximum reported mobility was slightly below $2000 \mathrm{~cm}^{2} /(\mathrm{V} \mathrm{s})$ [25], followed by theoretical prediction that optical phonons scattering mechanism limit is around $2000 \mathrm{~cm}^{2} /(\mathrm{V} \mathrm{s})$ [26]. The exception to this trend was given by our experiments on the GaN/AlGaN heterostructures grown on the bulk, low dislocation GaN substrates revealed mobility over $2500 \mathrm{~cm}^{2} /(\mathrm{V} \mathrm{s})$ [17, 27]. In Fig. 6 we present the RT mobility as a function of $2 \mathrm{DEG}$ concentration. Also, recently other groups demonstrated 2DEG mobility slightly above $2000 \mathrm{~cm}^{2} /(\mathrm{V}$ s) at RT [28-30].

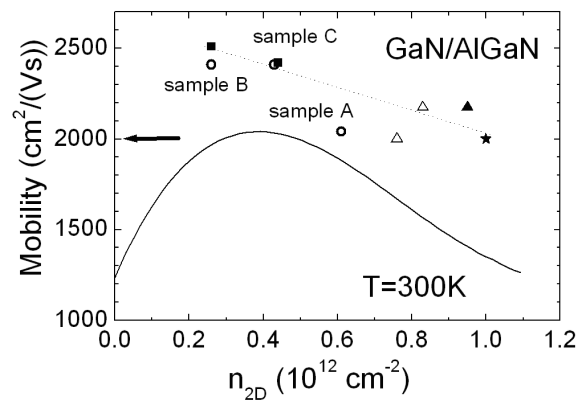

Fig. 6. The mobility dependence as a function of electron concentration of 2DEG on GaN/AlGaN interface at $300 \mathrm{~K}$. Circles and squares - the mobility derived from the Hall effect measurements and conductivity tensor analysis, respectively. Open triangles — Ref. [28], solid triangle - Ref. [29], star — Ref. [25], line - mobility of 2DEG on gated structure - Ref. [30]. Dashed line is the guide for the eye. Horizontal arrow points at the theoretical value of the optical phonon scattering limited mobility (after Ref. [26]).

The GaN/AlGaN heterostructures grown on bulk GaN crystals show also record low temperature mobility - above $100000 \mathrm{~cm}^{2} /(\mathrm{V} \mathrm{s})$ at $4 \mathrm{~K}$. The highest electron mobility measured in our samples are due to high quality of GaN/AlGaN interfaces, low donor/acceptor background doping (as was shown by analysis of photoluminescence (PL) on GaN layers) and low dislocation GaN bulk substrates. As we have recently shown [17, 27], samples with high electron mobility 2DEG enable fundamental studies of the quantum and many-body effects such as fractional quantum Hall effect, spin-related phenomena and magnetoexcitons.

\section{InGaN and AlGaN quantum wells}

One of the crucial elements in growth of blue-violet LEDs and LDs is optimization of the InGaN quantum wells by fine-tuning the growth parameters to 
maximize the room temperature PL intensity and to minimize full width at half maximum (FWHM) of the PL lines. We measured PL intensities of our multiquantum well (MQW) PAMBE structures and compared it with PL measured on reference MOVPE structure. The maximum PL for this test structure was around $\lambda=430 \mathrm{~nm}$ and our spectrometer and detector was calibrated to have a flat spectral response in the range $360-500 \mathrm{~nm}$. We find that peak PL intensities for our optimized test 10-period $\operatorname{In}_{x} \mathrm{Ga}_{1-x} \mathrm{~N} / \mathrm{In}_{0.02} \mathrm{Ga}_{0.98} \mathrm{~N}$ MQW structures grown on bulk GaN substrates are very comparable to the MOVPE-grown reference MQWs in the entire tested spectral range from 385 to $430 \mathrm{~nm}$ (see Fig. 7). Equally impor-

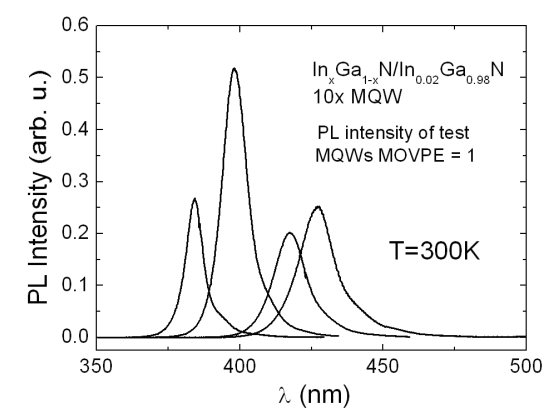

Fig. 7. Photoluminescence spectra of $\operatorname{In}_{x} \mathrm{Ga}_{1-x} \mathrm{~N} / \mathrm{In}_{0.02} \mathrm{Ga}_{0.98} \mathrm{~N}$ multiquantum wells at $T=300 \mathrm{~K}$.

tant are very small values for FWHM of the measured PL lines, varying from $7 \mathrm{~nm}$ (for PL line at $385 \mathrm{~nm}$ ) to $14 \mathrm{~nm}$ (for PL line at $430 \mathrm{~nm}$ ) with increasing In content in the QW from 5 to $17 \%$. The FWHM can be influenced by a number of factors, such as uniformity of In composition in the wells, uniformity of In composition from well to well and the quality of the interfaces. Figure 8 shows transmission electron microscopy image for $\operatorname{In}_{0.07} \mathrm{Ga}_{0.93} \mathrm{~N} / \mathrm{In}_{0.02} \mathrm{Ga}_{0.98} \mathrm{~N}$ single quantum well grown under such optimized conditions confirming the high compositional uniformity and excellent quality of the interfaces. In general, strong electric field present in nitride structures, grown on polar (0001) c-plane, changes PL energy and oscillator strength in QWs. To achieve high quantum efficiency from our InGaN QWs, the electric field was screened by Si doping of barriers. To give an insight on the role played by the built-in electric fields on performance of QWs we have grown GaN/AlGaN MQWs simultaneously on two different oriented substrates - on (0001) $c$-plane (which is typical polar orientation) and on (1120) $a$-plane (nonpolar orientation). Due to the absence of electric fields, growth of the QWs structures on nonpolar $a$-plane should lead to increase in PL intensity and change of its position in comparison with structures grown on polar direction. The bulk GaN substrates oriented in [1120] direction are available thanks to recent development of the hydride vapour phase epitaxy on bulk crystals at the Institute of High Pressure Physics, PAS [31]. The comparison of PL from AlGaN/GaN 10 MQWs (3 $\mathrm{nm}$ wells and $7 \mathrm{~nm}$ undoped barriers) grown on polar $c$-plane (dashes) and 


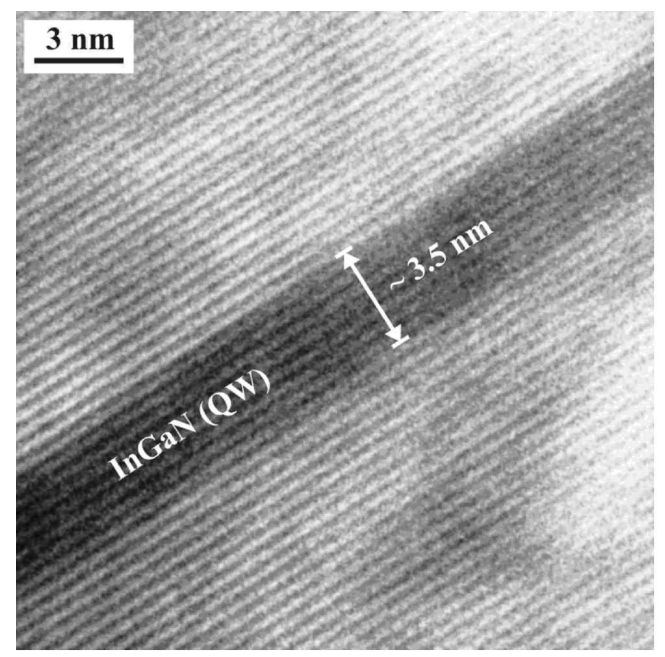

Fig. 8. High resolution transmission electron microscope image of $3.5 \mathrm{~nm}$ InGaN/InGaN single quantum well.

nonpolar $a$-plane (line) is shown in Fig. 9. The room PL energy for sample grown on nonpolar substrate is shifted to the higher energy and its intensity is more than $500 \times$ higher. In Fig. 10 the effect of electric field on QWs PL properties is schematically shown. The built-in electric field decreases the amplitude of PL (oscillator strength) and PL energy by spatial separation of electrons and holes. This effect becomes more pronounced for wider wells. Indeed - as it is shown in Fig. 11, for wider QWs grown on (0001) plane, strong quantum confined Stark effect (QCSE) is clearly observed (squares - experiment, dashed line - theory with electric field $E=500 \mathrm{keV} / \mathrm{cm}$ ). For QWs grown on nonpolar (1120) plane we observe quantum confinement (dots in Fig. 11) which can be easily predicted for standard rectangular well without electric field (solid line).

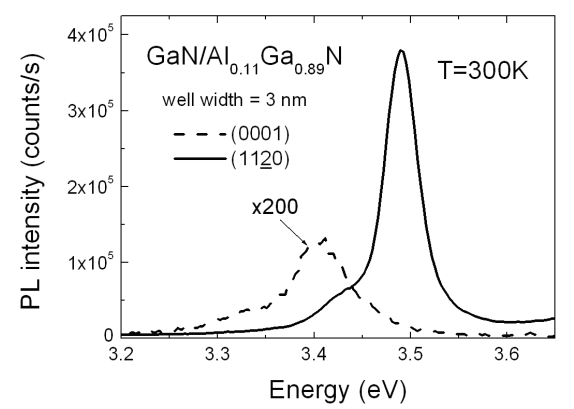

Fig. 9. Room temperature photoluminescence spectra of $\mathrm{GaN} / \mathrm{Al}_{0.11} \mathrm{Ga}_{0.89} \mathrm{~N}$ multiquantum wells grown on polar (dashed line) and nonpolar (solid line) orientations, respectively. 


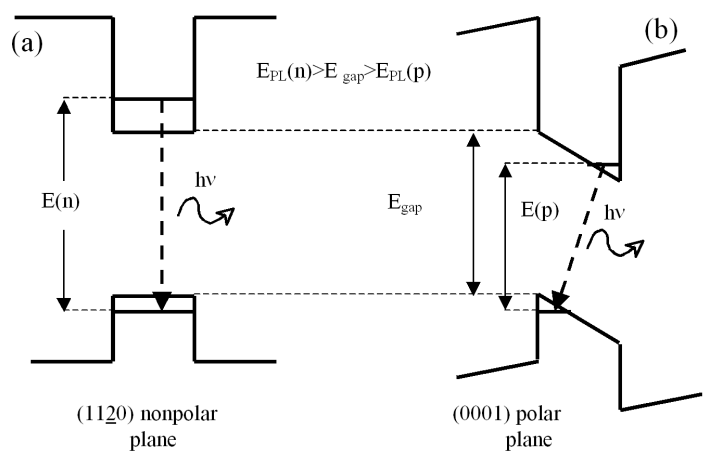

Fig. 10. Schematic diagram of optical transitions in quantum wells without (a) and with (b) electric field, respectively.

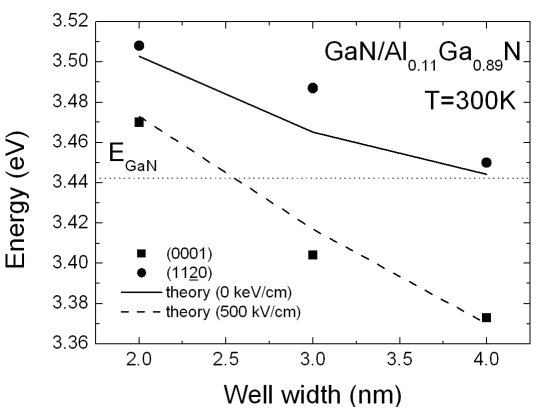

Fig. 11. The dependence of the photoluminescence energy as a function of well width for $\mathrm{GaN} / \mathrm{Al}_{0.11} \mathrm{Ga}_{0.89} \mathrm{~N}$ multiquantum wells grown on polar (squares) and nonpolar (dots) orientations, respectively. Solid and dashed line are calculations without and with $(500 \mathrm{kV} / \mathrm{cm})$ electric field, respectively. The dotted line indicates the energy gap of bulk GaN.

\section{Acceptor doping of GaN and InGaN}

Effective $p$-type doping of GaN is still a subject of intensive studies because it is crucial for nitride-based LEDs and LDs. The low electrical activation of Mg acceptor in GaN, due to its high ionization energy, requires very high doping level of $\mathrm{Mg}$ in order to obtain desirable hole concentrations. It was already reported that growth of GaN:Mg by PAMBE resulted in high $p$-type conductivity without any post growth annealing and with very sharp Mg doping profiles [32-37]. Systematic study of the Mg doping of GaN by Smorchkova et al. [32] showed that for the constant growth temperature, $\mathrm{Mg}$ flux controls $p$-type conductivity. The hole concentration as high as $1.3 \times 10^{18} \mathrm{~cm}^{-3}$ was achieved for $\mathrm{Mg}$ doping of $10^{20} \mathrm{~cm}^{-3}$. Growth at low temperatures increases $\mathrm{Mg}$ sticking coefficient considerably which can be an efficient method to achieve higher $\mathrm{Mg}$ doping level [33, 38]. However, for very high $\mathrm{Mg}$ flux, when the $\mathrm{Mg}$ surface coverage is about 1 monolayer (ML) the polarity inversion (PI) from Ga to $\mathrm{N}$ on GaN layer can take place [35-38] 
which leads to the abrupt decrease in the Mg doping efficiency and deteriorates dramatically crystal quality. Therefore the PI effect is a main limiting factor to achieve high $\mathrm{Mg}$ content in GaN layers.

The hole concentration was measured at the Hall bar geometry samples prepared by reactive ion etching on $0.5 \mu \mathrm{m}$ PAMBE GaN:Mg and InGaN:Mg layers grown on $\mathrm{GaN} / \mathrm{sapphire}$ templates. $10 \mathrm{~nm} / 20 \mathrm{~nm} \mathrm{Ni} / \mathrm{Au}$ contacts were annealed at $450^{\circ} \mathrm{C}$ for $10 \mathrm{~min}$ at oxygen atmosphere. The maximum measured hole concentration for GaN:Mg was $5 \times 10^{17} \mathrm{~cm}^{-3}$ with mobility $7 \mathrm{~cm}^{2} /(\mathrm{V} \mathrm{s})$ for $\mathrm{Mg}$ concentration $10^{20} \mathrm{~cm}^{-3}$ (see dots in Fig. 6). The $p$-type Hall conductivity depends on the $\mathrm{Mg}$ acceptor activation energy $E_{\mathrm{A}}$, number of $\mathrm{Mg}$ acceptors $N_{\mathrm{Mg}}$, and number of compensating donors $N_{\mathrm{D}}$. In Fig. 12 we plot the concentration of holes as a function of the $\mathrm{Mg}$ acceptors. Solid points show results of our experiments, while solid and dashed lines are calculations according to neutrality equation with acceptor ionization energy $E_{\mathrm{A}}=200 \mathrm{meV}$ and compensating donor concentrations $N_{\mathrm{D}}=0$ and $N_{\mathrm{D}}=10^{18} \mathrm{~cm}^{-3}$, respectively [38]. For modeling we use hole effective mass $m^{*}=1$.

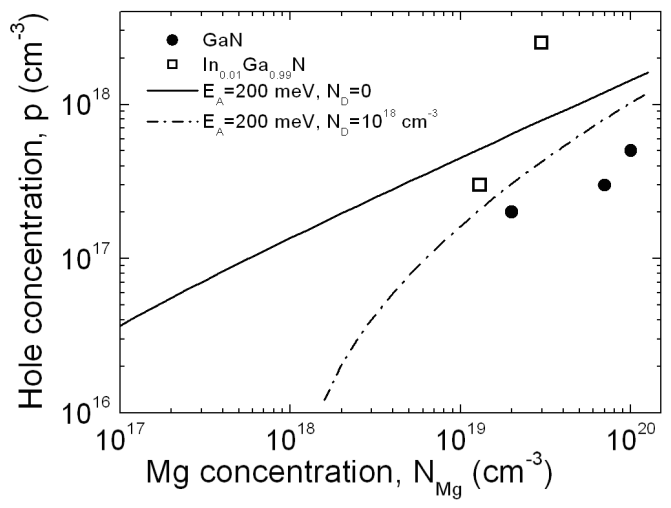

Fig. 12. The hole concentration as a function of the $\mathrm{Mg}$ acceptor content. Dots and open squares are experimental points for GaN and InGaN, respectively. Solid and dashed lines are calculations for acceptor activation energy $E_{\mathrm{A}}=200 \mathrm{meV}$, and compensating donor concentrations $N_{\mathrm{D}}=0$ and $N_{\mathrm{D}}=10^{18} \mathrm{~cm}^{-3}$, respectively.

There is a still animated discussion about the maximum $p$-type conductivity InGaN:Mg. Due to the lower hole effective mass in InGaN, the acceptor activation energy $E_{\mathrm{A}}$ should be smaller, which in turn should give more holes in valence band for the same doping level in comparison to GaN. In addition, localization effects present in InGaN can strengthen this trend. Indeed, in our PAMBE InGaN:Mg layers we observe substantial increase in the Hall concentration for the same Mg concentrations measured by secondary ion mass spectroscopy (SIMS) see squares in Fig. 12. The maximum Hall $p$-type concentration was found at the level $2-4 \times 10^{18} \mathrm{~cm}^{-3}$. 


\section{Blue-violet InGaN laser diodes}

The LDs structures consisted of the following sequence of layers (see Fig. 13). The $200 \mathrm{~nm}$ of GaN:Si buffer layer was followed by $0.55 \mu \mathrm{m}$ $\mathrm{Al}_{0.08} \mathrm{Ga}_{0.92} \mathrm{~N}: \mathrm{Si}$ cladding layer. The waveguide was asymmetric with lower part consisting of $100 \mathrm{~nm}$ GaN:Si and $40 \mathrm{~nm} \mathrm{In}_{0.01} \mathrm{Ga}_{0.99} \mathrm{~N}: \mathrm{Si}$. Depending on

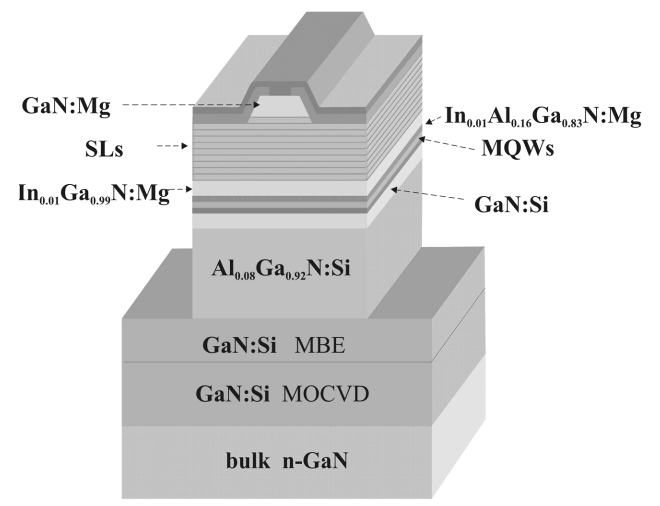

Fig. 13. Schematic structure of the PAMBE laser diode.

the structure, five, three or two undoped $4 \mathrm{~nm}$ thick $\operatorname{In}_{0.09} \mathrm{Ga}_{0.91} \mathrm{~N}$ QWs with $7 \mathrm{~nm} \operatorname{In}_{0.01} \mathrm{Ga}_{0.99} \mathrm{~N}: \mathrm{Si}$ barriers were deposited in the active region. $14 \mathrm{~nm}$ $\mathrm{In}_{0.01} \mathrm{Al}_{0.16} \mathrm{Ga}_{0.83} \mathrm{~N}: \mathrm{Mg}$ blocking layer was followed by $70 \mathrm{~nm} \mathrm{In}_{0.01} \mathrm{Ga}_{0.99} \mathrm{~N}: \mathrm{Mg}$ upper waveguide. The upper cladding consisted of 80 pairs of uniformly $\mathrm{Mg}$ doped $\mathrm{In}_{0.01} \mathrm{Ga}_{0.99} \mathrm{~N} / \mathrm{In}_{0.01} \mathrm{Al}_{0.16} \mathrm{Ga}_{0.83} \mathrm{~N}$ short period superlattice $(2.5 / 2.5 \mathrm{~nm})$. The contact layer was $100 \mathrm{~nm}$ GaN:Mg or InGaN:Mg. Doping levels for $n$-type layers was $5 \times 10^{18} \mathrm{~cm}^{-3}$, while hole concentration obtained from Hall effect measurements on a reference $0.3 \mu \mathrm{m}$ thick $\mathrm{In}_{0.01} \mathrm{Ga}_{0.99} N$ :Mg layer described in previous section was $2 \times 10^{18} \mathrm{~cm}^{-3}$. The growth temperature for GaN and AlGaN layers was $710^{\circ} \mathrm{C}$ (growth at Ga-rich regime), while QWs and $p$-type cladding were grown at $590^{\circ} \mathrm{C}$ (growth at In-rich regime). No post growth annealing was necessary to activate $p$-type conductivity. In Fig. 14 we show state of the art of the PAMBE technology - TEM picture of laser diode structure containing 3 InGaN/InGaN QWs. The laser diode devices were processed as ridge-waveguide, oxide-isolated lasers (Fig. 13). The mesa structures were etched out in the wafers down to a depth of $0.35 \mu \mathrm{m}$. The laser structures were then isolated by e-beam deposition of a $0.2 \mu \mathrm{m}$ layer of $\mathrm{SiO}_{x}$. The final devices had the stripe width of $15 \mu \mathrm{m}$ and the resonator length of $500 \mu \mathrm{m}$. The oxidized $\mathrm{Ni} / \mathrm{Au}$ ohmic contacts were deposited on the top surface of the devices, while Ti/Au contacts were deposited on the backside of the highly conducting $n$-GaN substrates. The tested devices had cleaved uncoated mirrors. 


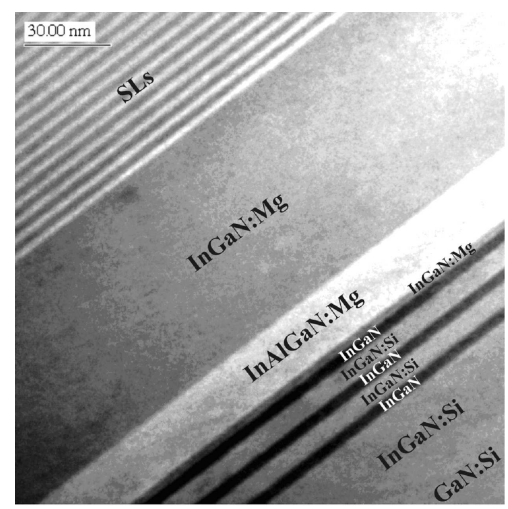

Fig. 14. Transmission electron microscope picture of 3 QWs laser diode structure.

The lasers operation was demonstrated at room temperature using $50 \mathrm{~ns}$ pulses at $0.25 \%$ duty cycle. Figure 15 presents light-current-voltage $(L-I-V)$ characteristics for our first 5 QWs LDs. A sharp increase in the light power visible at the current of about $1 \mathrm{~A}$ and $9 \mathrm{~V}$ bias is indicative of the laser operation. Above the threshold current strong narrowing of the spectral line was detected. As an example, in Fig. 15, we show spectra for 5 QWs LD. For this device multimode lasing was observed at $408 \mathrm{~nm}$ with mode spacing close to $0.53 \AA$ indicating the presence of longitudinal modes. The total line width of emission is close to $0.4 \mathrm{~nm}$. The lowest threshold current obtained for our lasers was $680 \mathrm{~mA}$ (at $8.2 \mathrm{~V}$ bias) which gives threshold current density of $9 \mathrm{kA} / \mathrm{cm}^{2}$ [39]. The slope efficiency per facet calculated from $L-I$ plot was $0.35 \mathrm{~W} / \mathrm{A}$ for $5 \mathrm{QWs}$ and $0.47 \mathrm{~W} / \mathrm{A}$ for $2 \mathrm{QWs}$ devices. The maximum output optical power was measured to be $0.9 \mathrm{~W}$ and $1.1 \mathrm{~W}$ for the LDs with 5 and 2 QWs, respectively. We would like to point out that threshold currents and voltages for 2 and 5 QWs LDs are very similar [19, 39]. The lasing operation was achieved in the wavelength range 408-422 $\mathrm{nm}$. The longer lasing wavelength was obtained by the increase in In content in quantum
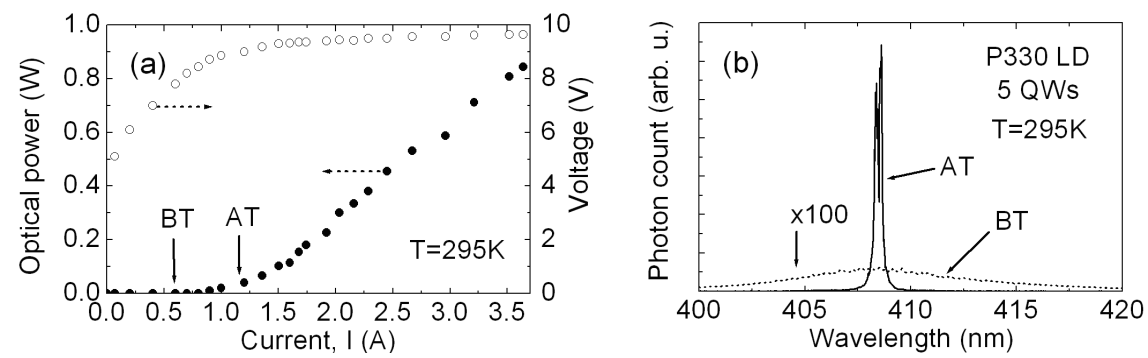

Fig. 15. (a) The light-current-voltage characteristic of 5 MQW laser diode. The arrows labeled BT and AT indicate the points where the emission spectra shown in part (b) were collected. (b) The emission spectra of the laser diode operating below (BT) and above lasing threshold (AT). 
wells. Since other device parameters are not changed, this result might indicate that only two quantum wells contribute to the lasing operation for the five QWs device, pointing towards possible structure design issues.

\section{Growth of strain compensated AlInN/InGaN MQWs}

By engineering the quantum confinement properties of electrons on a nanometer scale in $\mathrm{GaN} / \mathrm{Al}(\mathrm{Ga}, \mathrm{In}) \mathrm{N}$ heterostructures, it is possible to develop devices relying on intersubband (IS) absorption or emission at 1.3-1.55 $\mu \mathrm{m}$ telecom wavelengths. The operating principles of IS devices have already been successfully validated at mid- and far-IR wavelengths using semiconductor materials such as GaAs/AlGaAs or InGaAs/AlInAs-on-InP [40, 41]. The main technological problem in nitride based devices lies in the difference in lattice constants between GaN and AlN. The practical realization of GaN/AlN MQW devices leads to lattice relaxation and subsequent formation of cracks and large number of defects. Since InN has larger lattice constant than GaN, incorporation of In to AlN (in proportion $17 \% \mathrm{In}, 83 \% \mathrm{Al}$ ) gives InAlN lattice matched to GaN [42]. However, the band offset for such system is too small to reach $1.55 \mu \mathrm{m}$ IS absorption. Therefore we have grown barriers with higher Al content (93-96\%). To make full advantage of the GaN substrates we have grown strain compensated AlInN/InGaN MQWs. The $\mathrm{In}_{0.05} \mathrm{Ga}_{0.95} \mathrm{~N}$ quantum wells (instead $\mathrm{GaN}$ ) allowed us to achieve better strain compensation of $\mathrm{Al}_{0.966} \mathrm{In}_{0.04} \mathrm{~N} / \mathrm{In}_{0.05} \mathrm{Ga}_{0.95} \mathrm{~N}$ superlattices to GaN substrate. Structures with $20 \times$ QWs (with well width in the range 1.1-3 nm and barriers width of $3 \mathrm{~nm}$ ) grown on GaN/sapphire templates and bulk GaN crystals are crack-free, as demonstrated by the Nomarski contrast and scanning electron microscopic measurements. The performed X-ray diffraction (XRD) mapping of $a$ and $c$ constants show that AlInN/InGaN MQWs are fully strained - i.e. lattice constant $a$ of MQWs is equal to lattice constant $a$ of GaN substrate. We demonstrated IS absorption at room temperatures on these structures in the range $2.45-1.52 \mu \mathrm{m}$. The AlInN/InGaN strain compensated MQW structures grown by PAMBE on GaN substrates, thanks to the crackless growth and good optical quality, are very attractive for telecommunication applications at $1.5 \mu \mathrm{m}$ wavelengths like electro-optical modulators or all-optical switches. Due to the low value of the refractive index, AlInN/InGaN superlattices can also replace AlGaN/GaN in thick cladding layers in nitride optoelectronic devices e.g. in blue-violet laser diodes.

\section{Conclusions}

We show that making use of Ga and In surfactants, the high quality nitride devices can be grown by PAMBE at relatively low growth temperatures. The insight to the quality of the material obtained by this technology is given by properties of high mobility two-dimensional electron gas at GaN/AlGaN interface. 
The record mobility of 2DEG was obtained due to the low dislocation bulk substrates, high quality of interfaces and low residual background impurity doping level. Growth of efficient InGaN QWs together with high $p$-type doping levels in InGaN:Mg allowed us to demonstrate first blue-violet laser diodes. With continuing improvements in the quality of bulk GaN substrates and further optimization of the PAMBE growth conditions this technique is well positioned to become a viable alternative to the MOCVD technology in the near future.

\section{Acknowledgments}

This work was partially supported by Polish Government Program "The Development of Blue Optoelectronics" project No. 2700/C.T11-8/2000, by the project of the European Commission 6FP NITWAVE STREP 004170, and projects SPUM-M-NITWAVE of the Polish Ministry of Scientific Research and Information Technology.

\section{References}

[1] S. Nakamura, G. Fasol, The Blue Laser Diode, Springer, Heidelberg 1997.

[2] M. Kneissl, D.P. Bour, C.G. V.d. Walle, L.T. Romano, J.E. Northrup, R.M. Wood, M. Teepe, N.M. Johnson, Appl. Phys. Lett. 75, 581 (1999).

[3] N. Grandjean, B. Damilano, J. Massies, J. Phys., Condens. Matter 13, 6945 (2001)

[4] H. Morkoc, J. Mater. Sci., Mater. Electron 12, 677 (2001).

[5] T.D. Moustakas, E. Iliopoulos, A.V. Sampath, H.M. Ng, D. Doppalapudi, M. Misra, D. Korakakis, R. Singh, J. Cryst. Growth 227, 13 (2001).

[6] S.E. Hooper, M. Kauer, V. Bousquet, J.M. Barnes, J. Heffernan, Electron. Lett. 40, 33 (2004).

[7] J.A. Bardwell, Y. Liu, H. Tang, J.B. Webb, S.J. Rolfe, J. Lapointe, Electron. Lett. 39, 564 (2003).

[8] C.T. Foxon, T.S. Cheng, D. Korakakis, S.V. Novikov, R.P. Campion, I. Grzegory, S. Porowski, M. Albrecht, H.P. Strunk, MRS Internet J. Nitride Semicond. Res. 4S1, G4.11 (1999).

[9] H. Riechert, R. Averbeck, A. Graber, M. Schienle, U. Strauss, H. Tews, Mater. Res. Soc. Symp. Proc. 449, 149 (1997).

[10] J. Neugebauer, T.K. Zywietz, M. Scheffler, J.E. Northrup, H. Chen, R. M. Feenstra, Phys. Rev. Lett. 90, 056101 (2003).

[11] J.E. Northrup, J. Neugebauer, R.M. Feenstra, A.R. Smith, Phys. Rev. B 61, 9932 (2000).

[12] B. Heying, R. Averbeck, L.F. Chen, E. Haus, H. Riechert, J.S. Speck, J. Appl. Phys. 88, 1855 (2000).

[13] C.R. Elsass, I.P. Smorchkova, B. Heying, E. Haus, P. Fini, K. Maranowski, J.P. Ibbetson, S. Keller, P.M. Petroff, S.P. DenBaars, U.K. Mishra, J.S. Speck, Appl. Phys. Lett. 74, 3528 (1999). 
[14] C. Adelmann, J. Brault, D. Jalabert, P. Gentile, H. Mariette, G. Mula, B. Daudin, J. Appl. Phys. 91, 9638 (2002).

[15] M.J. Manfra, K.W. Baldwin, A.M. Sergent, K.W. West, R.J. Molnar, J. Caissie, Appl. Phys. Lett. 85, 5394 (2004).

[16] C. Skierbiszewski, Z. Wasilewski, M. Siekacz, A. Feduniewicz, B. Pastuszka, I. Grzegory, M. Leszczynski, S. Porowski, Phys. Status Solidi A 201, 320 (2004).

[17] C. Skierbiszewski, K. Dybko, W. Knap, J. Łusakowski, M. Siekacz, W. Krupczyński, G. Nowak, M. Boćkowski, Z. Wasilewski, D. Maude, T. Suski, S. Porowski, Appl. Phys. Lett. 86, 102106 (2005).

[18] P. Waltereit, H. Sato, C. Poblenz, D.S. Green, J.S. Brown, M. McLaurin, T. Katona, S.P. DenBaars, J.S. Speck, J.H. Liang, M. Kato, H. Tamura, S. Omori, C. Funaoka, Appl. Phys. Lett. 84, 2748 (2004).

[19] C. Skierbiszewski, Z. Wasilewski, M. Siekacz, A. Feduniewicz, P. Perlin, P. Wisniewski, J. Borysiuk, I. Grzegory, M. Leszczynski, T. Suski, S. Porowski, Appl. Phys. Lett. 86, 011114 (2005).

[20] W. Utsumi, H. Saitoh, H. Kaneko, T. Watanuki, K. Aoki, O. Shimomura, Nature Mater. 2, 735 (2003).

[21] J.A. Van Vechten, Phys. Rev. B 7, 1479 (1973).

[22] I. Grzegory, S. Porowski, Thin Solid Films 367, 281 (2000).

[23] I.P. Smorchkova, C.R. Elsass, J.P. Ibbetson, R. Vetury, B. Heying, P. Fini, E. Haus, S.P. DenBaars, J.S. Speck, U.K. Mishra, J. Appl. Phys. 86, 4520 (1999).

[24] L. Hsu, W. Walukiewicz, J. Appl. Phys. 89, 1783 (2001).

[25] R. Gaska, M.S. Shur, A.D. Bykhovski, A.O. Orlov, G.L. Snider, Appl. Phys. Lett. 74, 287 (1999).

[26] R. Oberhuber, G. Zandler, P. Vogl, Appl. Phys. Lett. 73, 818 (1998).

[27] M. Siekacz, K. Dybko, C. Skierbiszewski, W. Knap, Z. Wasilewski, D. Maude, J. Łusakowski, W. Krupczyński, G. Nowak, M. Boćkowski, S. Porowski, Phys. Status Solidi C 2, 1355 (2005).

[28] Z. Bougrioua, M. Azize, A. Jimenez, A.-F. Brana, P. Lorenzini, B. Beaumont, E. Muñoz, P. Gibart, Phys. Status Solidi C 2, 2424 (2005).

[29] M. Miyoshi, H. Ishikawa, T. Egawa, K. Asai, M. Mouri, T. Shibata, M. Tanaka, O. Oda, Appl. Phys. Lett. 85, 1710 (2004).

[30] M.J. Uren, T. Martin, B.T. Hughes, K.P. Hilton, A. Wells, R.S. Balmer, D.C. Herbert, A.M. Keir, D.J. Wallis, A.J. Pidduck, M. Missous, Phys. Status Solidi A 194, 468 (2002).

[31] B. Łucznik, B. Pastuszka, I. Grzegory, M. Boćkowski, G. Kamler, E. Litwin-Staszewska, S. Porowski, J. Cryst. Growth 281, 38 (2005).

[32] I.P. Smorchkova, E. Haus, B. Heying, P. Kozodoy, P. Fini, J.P. Ibbetson, S. Keller, S.P. DenBaars, J.S. Speck, U.K. Mishra, Appl. Phys. Lett. 76, 718 (2000).

[33] E. Haus, I.P. Smorchkova, B. Heying, P. Fini, C. Poblenz, T. Mates, U.K. Mishra, J.S. Speck, J. Cryst. Growth 246, 55 (2002).

[34] A.J. Ptak, T.H. Myers, L.T. Romano, C.G. Van de Walle, J.E. Northrup, Appl. Phys. Lett. 78, 285 (2001). 
[35] V. Ramachandran, R.M. Feenstra, W.L. Sarney, L. Salamanca-Riba, J.E. Northrup, L.T. Romano, D.W. Greve, Appl. Phys. Lett. 75, 808 (1999).

[36] L.T. Romano, J.E. Northrup, A.J. Ptak, T.H. Myers, Appl. Phys. Lett. 77, 2479 (2000).

[37] J.E. Northrup, Appl. Phys. Lett. 82, 2278 (2003).

[38] A. Feduniewicz, C. Skierbiszewski, M. Siekacz, Z. Wasilewski, I. Sproule, S. Grzanka, J. Borysiuk, G. Kamler, E. Litwin-Staszewska, R. Czernecki, M. Boćkowski, S. Porowski, J. Cryst. Growth 278, 443 (2005).

[39] C. Skierbiszewski, P. Perlin, I. Grzegory, Z.R. Wasilewski, M. Siekacz, A. Feduniewicz, P. Wisniewski, J. Borysiuk, P. Prystawko, G. Kamler, T. Suski, S. Porowski, Semicond. Sci. Technol. 20, 809 (2005).

[40] J. Faist, F. Capasso, D.L. Sivco, C. Sirtori, A.L. Hutchinson, A.Y. Cho, Science 264, 553 (1994).

[41] B.F. Levine, K.K. Choi, C.G. Bethea, J. Walker, R.J. Malik, Appl. Phys. Lett. 50, 1092 (1987).

[42] J.-F. Carlin, M. Ilegems, Appl. Phys. Lett. 83, 668 (2003). 